\title{
Die Schenkelhalsfraktur beim Kind
}

\author{
Wolfgang Schlickewei, Richard Salm
}

\section{Zusammenfassung:}

Die Schenkelhalsfraktur beim Kind ist eine seltene Verletzung. Im Gegensatz zu den einen osteoporotischen Knochen treffenden häufigen Verletzungen der Schenkelhalsregion des alten Patienten sind in der Regel hohe Krafteinwirkungen und direkte Traumamechanismen die auslösende Ursache. Das Behandlungskonzept wird durch die Besonderheiten der Blutversorgung des kindlichen Schenkelhalses und insbesondere seiner Epiphysenregion beeinflusst. Vor allem dislozierte Frakturen gefährden die Hüftkopf- durchblutung. Die Fraktureinteilung erfolgt nach einer Klassifikation von Delbet und Colonna. Als Behandlungskonzept hat sich die sofortige Reposition der Fraktur, die Entlastung des Gelenkhämatoms durch Eröffnung der Gelenkkapsel und das Stabilisieren der Fraktur mit kanülierten Schrauben bzw. bei wachstumsfugennahen Frakturen mit die Fuge kreuzenden Bohrdrähten bewährt. Die wesentliche Spätkomplikation der Verletzung, die durch Störung der Durchblutung bedingte Femurkopfnekrose, kann so in der überwiegenden Zahl der Fälle wirksam vermieden werden. und postnatalen Entwicklung des Gefäßsystems. Im Wesentlichen sind 3 Gefäßgruppen beteiligt:

Äste der A. circumflexa femoris medialis

- Äste der A. circumflexa femoris lateralis

Äste der A. obturatoria

Vor allem bei einer dislozierten Verletzung, aber auch bei unverschobenen Frakturen, sind Einblutungen in die straffe Gelenkkapsel nicht selten, die sekundär durch Druckerhöhung in der Hüftgelenkskapsel zu einer weiteren Durchblutungsstörung der gegebenenfalls beim Unfall nicht geschädigten Gefäße führen können.

\section{Besonderheiten der Anatomie des kindlichen Schenkelhalses}

Die spongiösen knöchernen Strukturen des kindlichen Schenkelhalses sind sehr hart, so dass erhebliche Kräfte zum Entstehen einer Fraktur notwendig sind. Darüber hinaus besteht die Besonderheit der Blutversorgung, die über die Schenkelhalsregion umspannende Blutgefäße erfolgt. Währenddessen hat die Blutversorgung der Epiphyse über das Ligamentum teres capitis femoris quantitativ keine Bedeutung.

Zur Gefäßversorgung der Schenkelhalsregion (Abb.1) beim Kind liegen zahlreiche Untersuchungen vor. Trueta [10] hat bereits vor etwa 50 Jahren in ausgedehnten anatomischen Untersuchungen diese Besonderheiten heraus gearbeitet, die für das weitere Behandlungskonzept von wesentlicher Bedeutung sind. Batory [1] hat 1982 Studien vorgestellt zur prä-

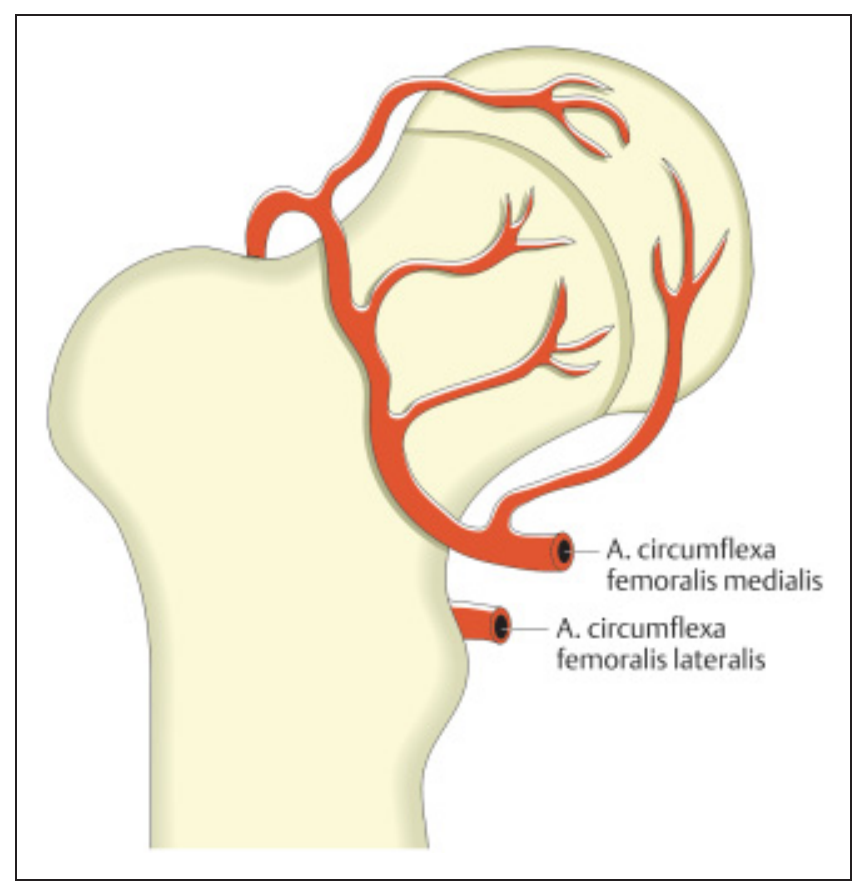

Abb. 1 Arterielle Versorgung des Hüftkopfes. Die Gefäßäste aus der Arteria circumflexa femoris medialis und lateralis umgehen die Wachstumsfuge intra- und extrakapsulär. 


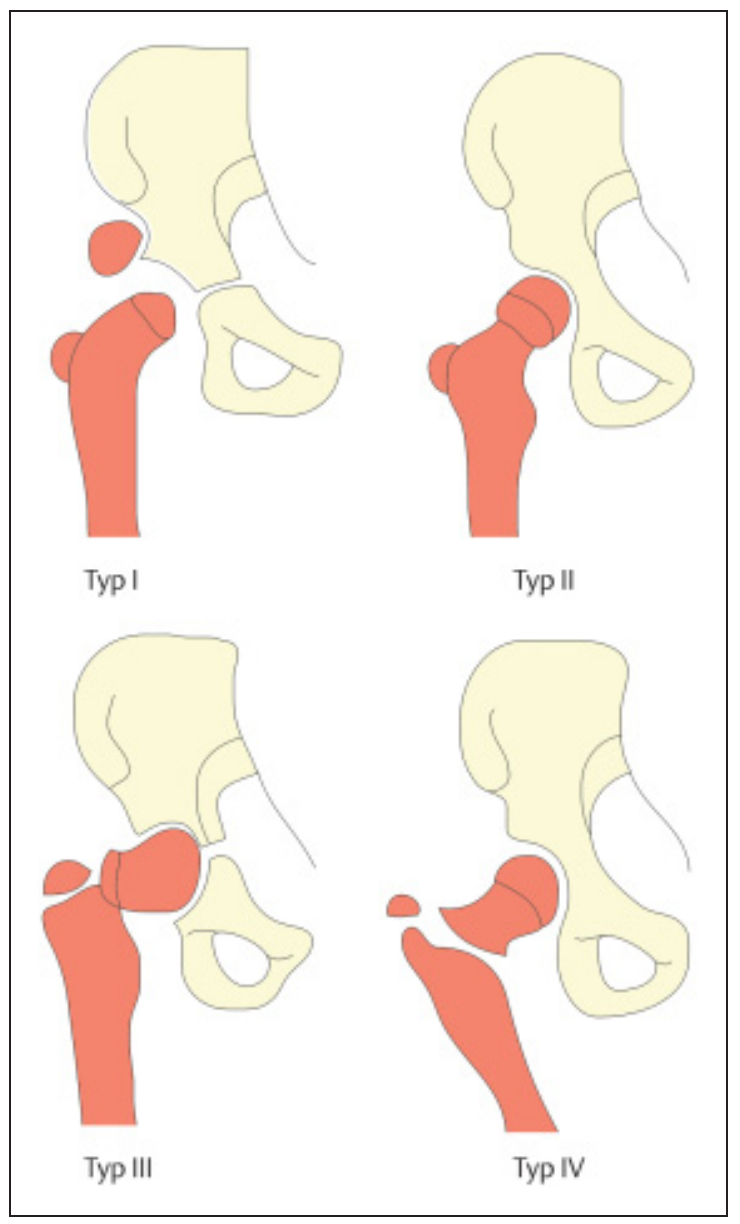

Abb. 2 Klassifikation der medialen Schenkelhalsfrakturen im Kindesalter: (nach Delbet und Colonna):

Typ I: transepiphysär

Typ II: transzervikal

Typ III: zervikotrochantär

Typ IV: intertrochantär

\section{Epidemiologie der Verletzung}

Zur Schenkelhalsfraktur bei Kindern und Jugendlichen gibt es verschiedene Häufigkeitsangaben. Insgesamt macht die Schenkelhalsfraktur im Wachstumsalter weniger als $1 \%$ der Verletzungen an der unteren Extremität aus. Dies bedeutet, dass auch in spezialisierten Kliniken, die eine große Zahl von Kinderfrakturen behandeln, im Durchschnitt nur ein Kind im Jahr zur Behandlung kommt.

Niethart [6] hat in einer Sammelpublikation über 700 Fälle aus insgesamt 38 zum Thema vorliegenden Publikationen ausgewertet. Epidemiologisch handelt es sich hier vor allem um Frakturen vom Typ II und Typ III (je $42 \%$ der erfassten Patienten), die Verletzungen von Typ I und Typ IV nach Delbet und Colonna wurden in je $8 \%$ der Fälle beobachtet.

In einer eigenen Fallserie konnten konsekutiv 28 Fälle aus 20 Jahren, die in der Unfallabteilung der Universitätsklinik Freiburg zur Behandlung kamen, beobachtet und ausgewertet werden $[8,9]$. In diesem Kollektiv wurde bei 17 der 28 Patienten eine Fraktur vom Typ III (laterale Schenkelhalsfraktur) behandelt, die bei Kindern in Relation häufiger als beim Erwachsenen beobachtet wird.

\section{Klinik und Diagnostik}

Die klinische Symptomatik besteht vor allem in schmerzhaft eingeschränkter Beweglichkeit im Hüftgelenk. Die beim alten Patienten häufig beobachtete Außenrotation und Verkürzung des Beines wird bei Schenkelhalsfrakturen der Kinder nicht so regelhaft beobachtet, da selten massive Dislokationen vorliegen. Die Diagnosestellung ist durch die konventionelle Beckenübersichtsaufnahme und gegebenenfalls eine axiale bzw. Lauensteinstellung angefertigte Aufnahme in der Regel ohne Probleme möglich. Bei unsicherer radiologischer Befundsituation und entsprechender Klinik sollte nicht zuletzt aus Strahlenschutzgründen als ergänzende Untersuchung heute eine Kernspinuntersuchung (keine computertomographische Untersuchung) des kindlichen Schenkelhalses erfolgen, um so die Strahlenbelastung entsprechend zu reduzieren.

\section{Behandlungskonzept}

Die Schenkelhalsfraktur beim Kind stellt wegen der gefährdeten Durchblutungssituation durch die Fraktur selbst und führen soll; sie ist derzeit noch in Validierung.
Typ II:

kethatsf wie beim Typ I, um eine intraartikuläre Verletzung.
Typ III:

Die Internationale Arbeitsgemeinsc Osteosynthesefragen (AO-I) ent von Maurice Müller [5] entwickelte Erwachsenen-Klassifikation das Prinzip der ABC-Klassifikation in modifizierter Form auch für kindliche Frakturen ein- 


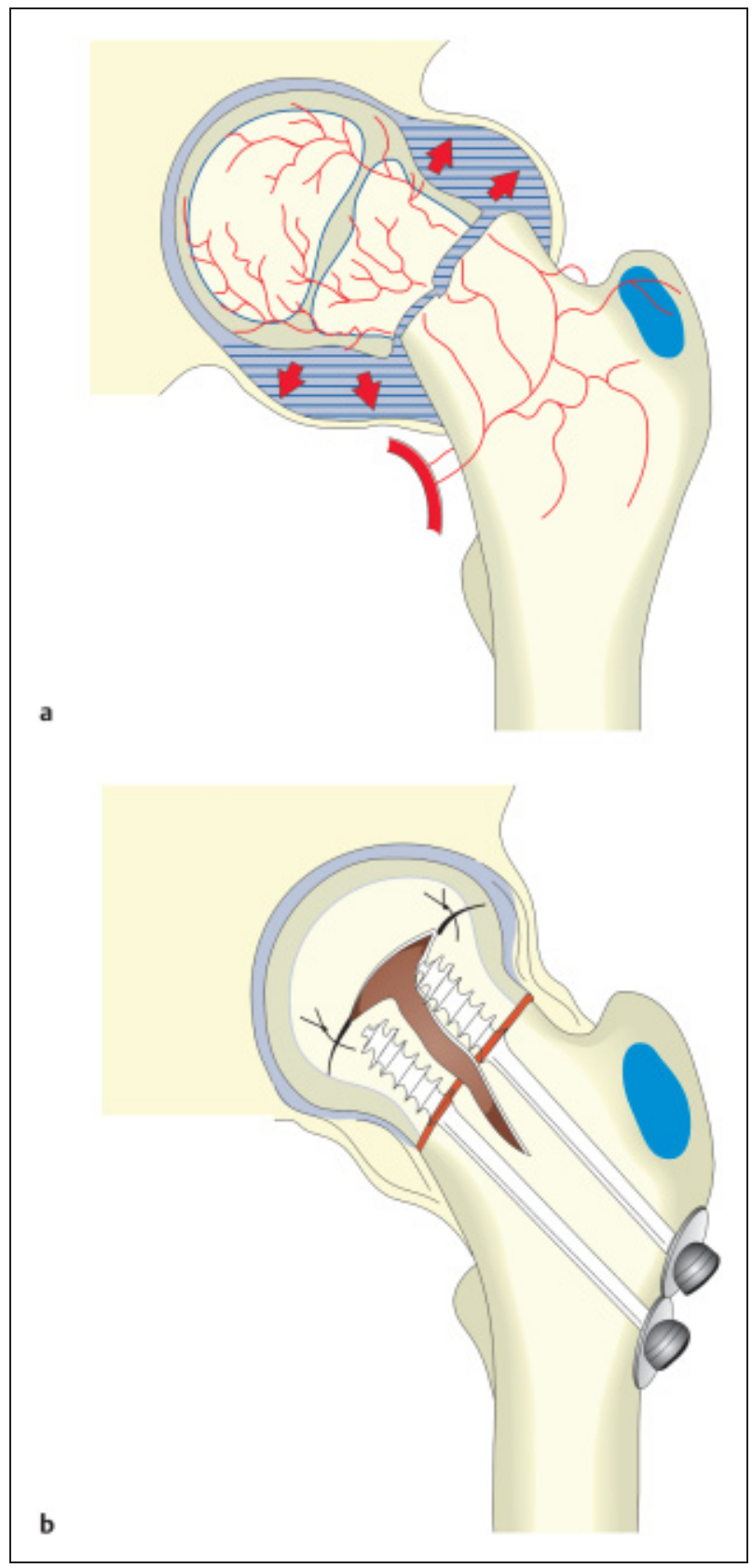

durch das sekundär drohende Spannungshämarthros (Abb. $\mathbf{3}$ ) einen chirurgischen Notfall dar $[8,9,11]$. Anderslautende Mitteilungen sollten mit Vorsicht interpretiert werden [4]. Mit Diagnosestellung ist bei dislozierter Fraktur die Indikation zum operativen Vorgehen gegeben. Die Versorgung sollte notfallmäßig erfolgen, mit dem Ziel der Entlastung des Spannungshämarthros und einer übungsstabilen Versorgung der Fraktur.

Ein konservatives Vorgehen ist ausschließlich gestattet bei nicht dislozierter Fraktur und sonographisch kontrolliertem Hüftgelenk mit Ausschluss eines
Abb.3a Spannungshämarthros nach intrakapsulärer medialer Schenkelhalsfraktur beim Kind

Abb. $\mathbf{3 b}$ OP Konzept: ventrale Fensterung der Kapsel und Stabilisierung mit Spongiosaschrauben mit kurzem Gewinde
Frakturtisch ohne Extension erfolgen, um intraoperativ die korrekte anatomische Reposition der Fraktur zu erreichen.

- Notfallindikation

- Entlastung des Spannungshämarthros

- Frakturreposition

- Unmittelbare operative übungsstabile Versorgung

\section{Osteosyntheseverfahren}

Bei epiphysären Verletzungen wird analog zur Epiphyseolyse des präpubertären Kindes eine Stabilisierung der Dislokation durch Frakturspalt überbrückende Bohrdraht-Osteosynthese durchgeführt. Der Bohrdraht kann hier, abhängig vom Alter des Kindes im Durchmesser von 1,8 bis $2,5 \mathrm{~mm}$ gewählt werden. In der Regel sind 3 bis subkortikal unter die Gelenkebene eingebrachte Bohrdrähte, die an der lateralen Kortikalis umgebogen und gekürzt werden, zur Stabilisierung ausreichend. Gewindebohrdrähte können die Retention der Fraktur in der subchondralen Kortikalis besser gewährleisten

Bei der Mehrzahl der Typ-II- und bei allen Typ-III-Frakturen kann die Versorgung mit Spongiosaschrauben (Abb.4) mit kurzem Gewinde gewählt werden, wobei die Gewindegänge so positioniert werden, dass sie jenseits der Frakturebene zu liegen kommen und gleichzeitig die Wachstumsfuge nicht tangieren. Gegebenenfalls können die Spongiosaschrauben im Gewindeanteil gekürzt werden. Die Verwendung der kanulierten Schraubenimplantationstechnik vereinfacht die Schraubenpositionierung wesentlich. Darüber hinaus gewährleistet sie ein paralleles Einbringen der Schrauben in allen drei Ebenen, so dass bei leichter Sinterung in der Frakturzone gegebenenfalls ein Kompressionsmechanismus gewährleistet bleibt. $\mathrm{Zu}$ beachten ist, dass bei Kindern und Jugendlichen aufgrund der harten Spongiosa ein Gewindeschneiden unbedingt indiziert ist. Darüber hinaus sollten beim Einbringen der ersten Schraube mindestens 2 weitere Bohrdrähte eingebracht sein, um einen Antirotationsschutz beim Schneiden des $\mathrm{Ge}$ windes und beim Einbringen der Schraube zu gewährleisten. Je nach Größe des Schenkelhalses können 2 bis 3 Schrauben positioniert werden. Die Größenwahl (Durchmesser) ist abhängig vom Alter des Kindes. Bei jüngeren Kindern sind Kleinfragment-, bei älteren Kindern in der Regel Großfragmentschrauben das Implantat der Wahl. 

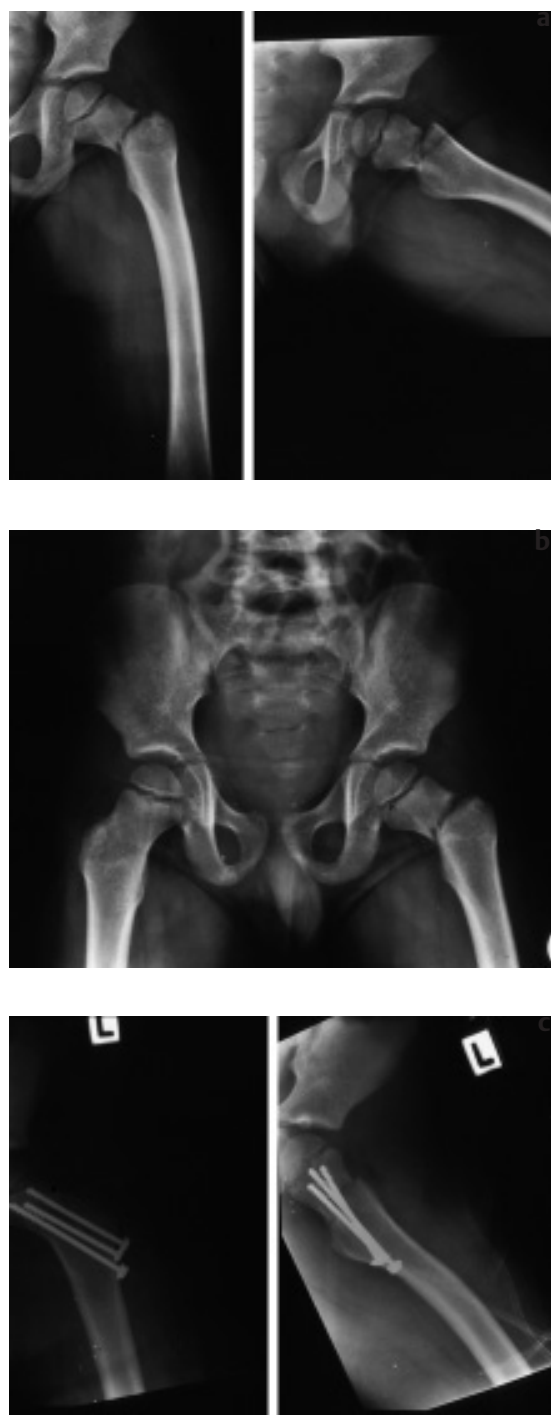

Abb.4a-c Schenkelhalsfraktur bei 6jährigem Jungen - Sturz beim Skifahren (direktes Trauma). Unfallaufnahmen (a, b), Versorgung mit Kleinfragment-Spongiosaschrauben mit kurzem Gewinde (c).

Bei der pertrochantären Fraktur im Kindesalter muss abhängig von der Fraktursituation und vom Frakturverlauf entschieden werden. Hier kann die Kombination einer Schraubenosteosynthese mit einer Trochanter-Zuggurtung, die distal am Femur an einer Schraube aufgehängt wird, zu einer suffizienten Stabilisierung führen. In Einzelfällen ist auch die Verwendung einer in kanülierter Technik einzubringenden Winkelplatte, wie sie bei der Oberarmkopffraktur des Erwachsenen verwendet werden kann, sinnvoll.

Eine zusätzliche Ruhigstellung, gegebenenfalls im Beckenbeingips, ist dann anzustreben, wenn aufgrund des Alters (Kleinkind) oder bei fehlender Kooperation eine passagere Sicherung der Osteo-

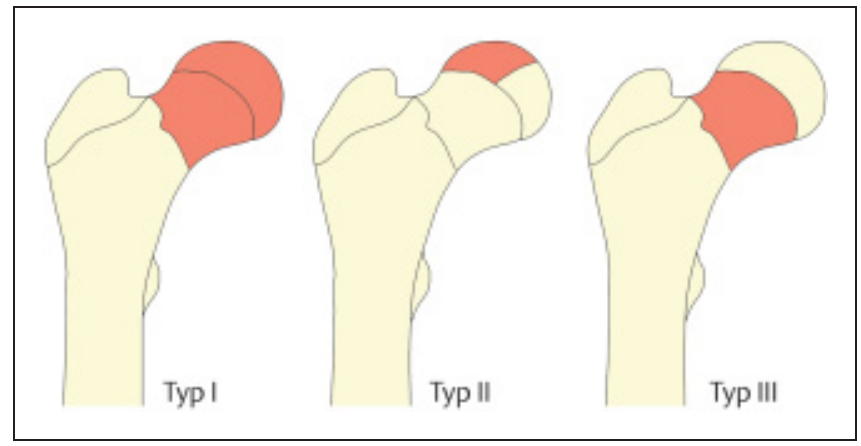

Abb.5 Formen der avaskulären Hüftkopfnekrose beim Kind (Einteilung nach Ratliff).

synthese in den ersten Tagen erfolgen sollte. Die Ruhigstellung kann aber nach unserer Erfahrung auf 3 Wochen begrenzt werden.

- Bei epiphysären Frakturen fugenspaltüberbrückende Bohrdrahtosteosynthese (Frakturtyp I)

- Bei Frakturen von Typ II und Typ III Versorgung mit (kanülierten) Spongiosaschrauben ohne sekundäre Schädigung der Wachstumsfuge

- Bei Frakturen vom Typ IV Trochanterzuggurtung in Kombination mit Schraubenosteosynthese, gegebenenfalls kanülierte Winkelplatte.

\section{Prognose und Komplikationen}

Die Prognose der Verletzung ist bei konsequenter Einhaltung des Therapiekonzepts gut. Im eigenen Kollektiv konnten wesentliche Spätkomplikationen, vor allem die Femurkopfnekrose, bei den nach diesem Konzept behandelten Fällen regelhaft vermieden werden. Die wesentliche Spätkomplikation bleibt die avaskuläre Hüftnekrose (Abb.5), bei der verschiedene Nekroseformen nach Ratliff [7] zu unterscheiden sind (Fallbeispiel Abb.6). Hier kann es sich entweder um Nekrosetypen vom
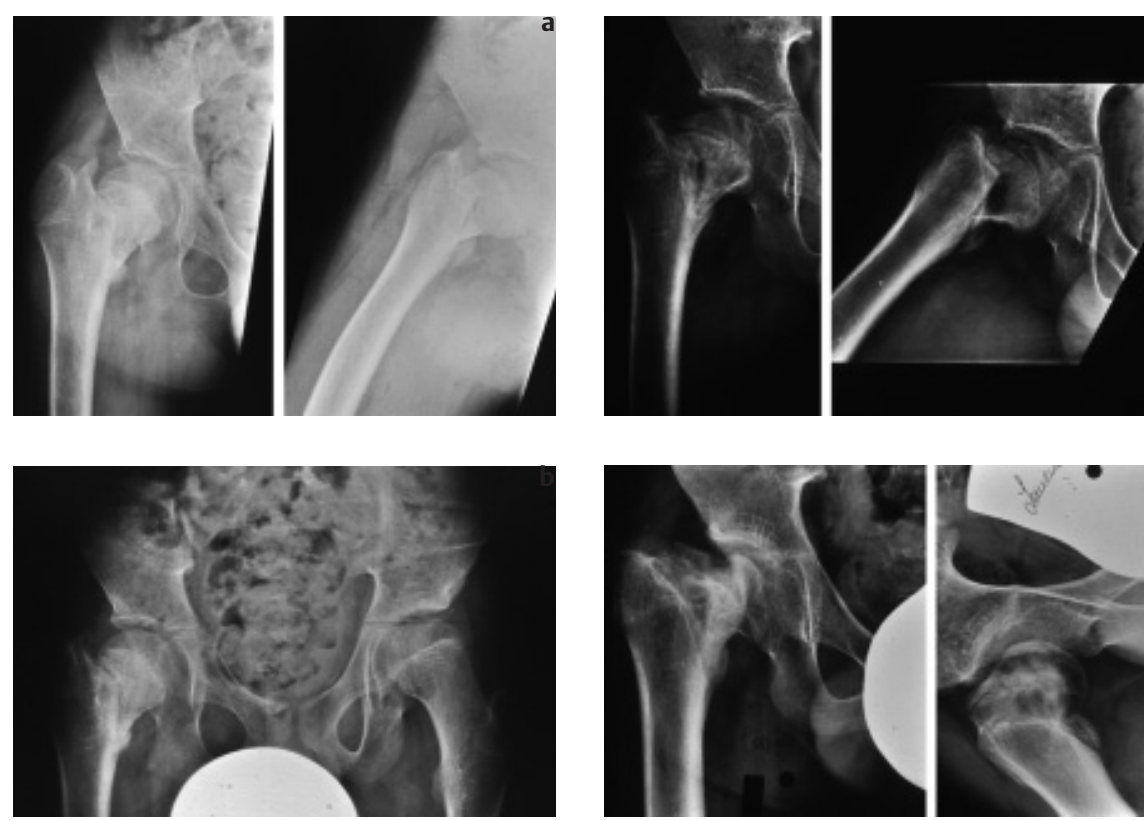

Abb.6a-d Spontanverlauf einer unbehandelten lateralen Schenkelhalsfraktur bei behindertem 9-jährigen Jungen. Primär behandelt als Hüftgelenksprellung, Diagnosestellung nach 4 Wochen. Ablehnung einer operativen Intervention durch die Eltern. Der Junge hat im weiteren Verlauf weiter belastet. Die abschließende Verlaufskontrolle nach $1 \frac{1}{2} 2$ Jahren zeigt die typische Hirtenstab-Deformität des mit partieller Hüftkopfnekrose verheilten rechten Schenkelhalses. Bei Beinlängendifferenz von $2 \mathrm{~cm}$ ist der Junge subjektiv zu diesem Zeitpunkt noch weitgehend beschwerdefrei. 
Typ I: Kopf- und Halsnekrose, vom Typ II: partielle, isolierte Kopfnekrose und

Typ III: partielle, isolierte Halsnekrose handeln.

Die Ausbildung einer Pseudarthrose ist in der spongiösen Knochenstruktur des Kindes auch in dieser Region beim Kind eine absolute Rarität.

\section{Nachbehandlungskonzept}

In der postoperativen Behandlung ist eine Entlastung unter Mobilisation an Unterarmgehstöcken mit Fußsohlenbodenkontakt bis zur sicheren knöchernen Konsolidierung der Frakturzone zu gewährleisten. Dies ist in der Regel nach 6 bis 8 Wochen zu beobachten. Bei jüngeren Kindern kann gegebenenfalls bei fehlender Kooperation eine passagere Ruhigstellung im Becken-Bein-Gips indiziert sein.

Die Implantatentfernung kann nach sicherer knöcherner Konsolidierung erfolgen. Dies ist in der Regel nach 9 Monaten möglich. Im weiteren Verlauf sollte das Wachstum bis zum Fugenschluss beobachtet und klinisch kontrolliert werden. Nur in besonderen Verdachtsmomenten sind gegebenenfalls auch radiologische Verlaufskontrollen im Jahresabstand, die gegebenenfalls zur Reduzierung der Strahlenbelastung in kernspintomographischer Technik erfolgen können, erforderlich.

\section{Literatur}

${ }^{1}$ Batory I. Die Bedeutung der Gefäßentwicklung für die Ossifikation der proximalen $\mathrm{Fe}$ murepiphyse. Z Orthop 1982; 120: 690

${ }^{2}$ Colonna PC. Fracture of the neck of the femur in children. Am J Surg 1929; 6: 793

${ }^{3}$ Delbet P. zitiert in Colonna (1929)

${ }^{4}$ May J, Hirner V, Styhler W, Posch E, Jelen M, Linhart WE, Kohlmaier W, Neubauer T, Schwarz N. Schenkelhalsfrakturen im Kindesalter. Unfallchirurg 1998; 101: 426

${ }^{5}$ Müller ME, Nazarian S, Koch P, Schatzker J. The comprehensive classification of fractures of long bones. Springer, Berlin (1990)

${ }^{6}$ Niethard FU. Pathophysiologie und Prognose von Schenkelhalsfrakturen im Kindesalter. Hefte Unfallh 1982; 158: 221

${ }^{7}$ Ratliff AHC. Fracture of the neck of the femur in children. J Bone Joint Surg [Br] 1962; 44: 528
${ }^{8}$ Schlickewei W, Paul Ch. Behandlungskonzept bei kindlichen Schenkelhalsfrakturen. Hefte Unfallh 1993; 228: 27

${ }^{9}$ Schlickewei W, Seif el Nasr M, Breckwoldt M, Friedl HP. Therapierichtlinien und Ergebnisse bei Schenkelhalsfrakturen im Kindesalter. Kongressband Dt. Gesellschaft für Chirurgie 1999; 519

${ }^{10}$ Trueta J. The normal vascular anatomy of the human femoral head during growth. J Bone Joint Surg [Br] 1957; 39: 358

${ }^{11} \mathrm{v}$. Laer L. Frakturen und Luxationen im Wachstumsalter. Thieme, Stuttgart 2001

Prof. Dr. med. Wolfgang Schlickewei Chefarzt Unfallchirurgie

Priv.-Doz. Dr. med. Richard Salm Chefarzt Allgemein-Chirurgie

Chirurgische Klinik Abteilung Unfallund Wiederherstellungschirurgie im Regionalverbund kirchlicher Krankenhäuser,

St. Josefskrankenhaus,

Sautierstraße 1

D-79104 Freiburg 